\section{Comment on "Conductance Oscillations Periodic in the Density of a One-Dimensional Electron Gas"}

In a recent Letter, Scott-Thomas et al. ${ }^{1}$ announced the experimental discovery of conductance oscillations periodic in the density of a narrow Si inversion layer. An interpretation in terms of pinned charge-density waves was suggested. ${ }^{1,2}$ We propose an alternative single-electron explanation of this remarkable effect, based upon the concept of the Coulomb blockade of tunneling (arising from the charging energy associated with the tunneling of a single electron). Likharev ${ }^{3}$ and Amman, Mullen, and Ben-Jacob ${ }^{4}$ have studied theoretically the possibility of removing the Coulomb blockade by capacitive charging (by means of a gate terminal) of the region between two tunnel junctions in series. They found that the zero-bias conductance of such a device exhibits periodic peaks as a function of gate voltage, due to the modulation of the charging energy. We propose that the current through the channel in Ref. 1 is limited by tunneling through potential barriers constituted by two dominant scattering centers which delimit a segment of the one-dimensional channel (see Fig. 1). We describe the two tunnel barriers by capacitances $C_{1}$ and $C_{2}$. Because the number of electrons localized in the region between the two barriers is necessarily an integer, a charge imbalance, and hence an electrostatic potential difference, arises between this region and the adjacent regions connected to wide-electron-gas reservoirs. As the gate voltage is varied, the resulting Fermi-level difference $\Delta E_{F}$ oscillates in a sawtooth pattern between $\pm e \Delta$, where $\Delta=e / 2 C$ is the voltage drop over the effective capacitance $C=C_{1}+C_{2}$ with charge $e / 2$. The single-electron charging energy $e^{2} / 2 C$ maintains the Fermi-level difference, until $\Delta E_{F}= \pm \Delta$. Then the energy for the transfer of a single electron to (or from) the region between the two barriers vanishes, so that the Coulomb blockade is removed, and the conductance shows an unactivated maximum at low temperatures $T$ and source-drain voltages $V\left(k_{B} T / e, V \lesssim \Delta\right) .^{3,4,5}$ The oscillation of the Fermi energy as the gate voltage is varied thus leads to a sequence of conductance peaks. The periodicity of the oscillations corresponds to the addition of a single electron to the region between the two scattering centers forming the tunnel barriers, so that the oscillations are periodic in the density- as in the experiment. This single-electron tunneling mechanism also explains the observed activation of the conductance minima, and the insensitivity to a magnetic field. ${ }^{1,2}$ The capacitance

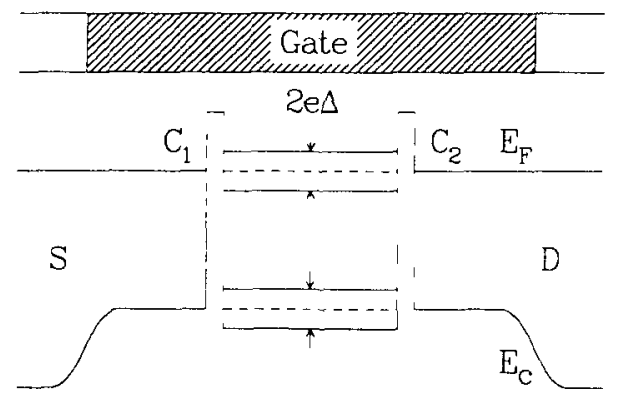

FIG. 1 Schematic representation of the bottom of the conduction band $E_{c}$ and Fermi energy $E_{F}$ in the device of Ref. 1 along the channel. The band bending at the connections of the narrow channel to the wide source $S$ and drain $D$ regions arises from the higher threshold for the electrostatic creation of an inversion layer by a narrow gate (shaded part). Tunnel barriers associated with two scattering centers are shown. The maximum Fermi-energy difference $\Delta E_{F}= \pm e \Delta$ [with $\Delta=e$ ] $2\left(C_{1}+C_{2}\right)$ ] sustainable by the Coulomb blockade is indicated.

associated with the scattering centers is hard to ascertain, but the experimental value of the activation energy, $\Delta E \approx 50 \mu \mathrm{eV}$, yields $C \approx e^{2} / 2 \Delta E \approx 10^{-15} \mathrm{~F}-$ a value typical for observations of the Coulomb blockade. ${ }^{3,4}$ To our knowledge, the idea that a Coulomb blockade may be associated with scattering centers in a onedimensional electron gas, acting as tunnel barriers with a small capacitance, has not been suggested before.

\section{H. van Houten and C. W. J. Beenakker \\ Philips Research Laboratories \\ 5600 JA Eindhoven, The Netherlands}

Received 26 June 1989

PACS numbers: 73.20.Dx, 71.45.Lr, 72.15. Nj

1J. H. F. Scott-Thomas, S. B. Field, M. A. Kastner, H. I. Smith, and D. A. Antoniadis, Phys. Rev. Lett. 62, 583 (1989).

${ }^{2}$ The same interpretation has been given to a similar effect in GaAs by U. Meirav, M. A. Kastner, M. Heiblum, and S. J. Wind, Phys. Rev. B 40, 5871 (1989).

${ }^{3}$ K. K. Likharev, I.B.M. J. Res. Dev. 32, 144 (1988), and references therein.

${ }^{4}$ M. Amman, K. Mullen, and E. Ben-Jacob, J. Appl. Phys. 65, 339 (1989); see also L. I. Glazman and R. I. Shekhter (unpublished).

${ }^{5}$ In the case of very different tunneling rates through the two barriers, one would expect steps in the current as a function of source-drain voltage, which are not observed in Ref. 1. For two similar barriers this "Coulomb staircase" is suppressed (see, e.g., Fig. 3 in Ref. 4). 
Kastner et al. Reply: van Houten and Beenakker ${ }^{1}$ (vHB) propose an interesting model, based on the idea of tunneling modulated by a Coulomb blockade (CB), to explain our recent experiments on narrow electron gases in $\mathrm{Si}$ inversion layers ${ }^{2}$ and pinched GaAs channels. ${ }^{3}$ The energy scale of this blockade is $e^{2} / 2 C$, where $C$ is the effective capacitance of one of the barrier junctions. We argue here that this capacitance is known, and leads to an energy scale higher than that measured in our experiments. We also point out that the simple relationships between measured quantities, required by the CB model, are not observed experimentally. The pinned chargedensity wave or Wigner-crystal model proposed in Refs. 2 and 3 does not suffer from these difficulties.

Within the CB model one expects the conductance to be thermally activated with an activation energy close to $\Delta E-e^{2} / 2 C$. vHB thus argue that our experimental activation energy $\Delta E \approx 50 \mu \mathrm{eV}$ implies a capacitance across the barriers of some $10^{-15} \mathrm{~F}$. They claim that the true value of the capacitance is difficult to ascertain, but that $10^{-15} \mathrm{~F}$ is not unreasonable. We contend that the relevant capacitance, namely, that of the segment isolated by barriers, can be estimated with some accuracy, and that its value is too small to explain the measured activation energy. The direct capacitance across the barrier is, indeed, hard to estimate, but it is certainly very small: Two electrodes of area $25 \times 5 \mathrm{~nm}^{2}$ (the inversion layer cross section) $10 \mathrm{~nm}$ apart (they cannot be much closer) have $C$ only $-10^{18} \mathrm{~F}$. There is, however, a much larger effective capacitance associated with the parallel capacitances of each side of the barrier to the top and bottom gates. The latter has the larger capacitance of the two, about $3 \mathrm{pF} / \mathrm{cm}$, so that $C \$ 10^{-16} \mathrm{~F}$ for a segment of typical length $L_{0}$, namely, several hundred nanometers. This is the largest capacitance associated with the segment, but it is still an order of magnitude smaller than the value needed by vHB. The charging energy associated with a $10^{-16} \mathrm{~F}$ capacitance is $\Delta E=800$ $\mu \mathrm{eV}$, which is difficult to reconcile with the $50-\mu \mathrm{eV}$ experimental value.

A remarkable feature of the Coulomb-blockade model is that the threshold voltage for nonlinear conduction $V_{T}$ is predicted to equal $\Delta E / e-e / 2 C$. In the two cases where both these parameters were measured, we find that $V_{T} \approx 200 \mu \mathrm{V}$ is about 4 times $\Delta E / e \approx 60 \mu \mathrm{V}$. It is not clear whether this discrepancy is conclusive evidence against the CB model, and more measurements are under way that should resolve this issue.
In Refs. 2 and 3 it was suggested that the ground state of these one-dimensional electron systems is, in fact, a Wigner crystal or charge-density wave. Such a wave is pinned by a small number of interface charges along the narrow channel. In this picture, the pinning energy is periodic in the gate voltage irrespective of whether the pinning centers are strong enough to constrain the number of wavelengths between two pinning centers to be an integer. The activation energy and the threshold field are both related to the strength of the pinning, but there is no prediction that $\Delta E, e V_{T}$, and $e^{2} / 2 C$ are equal. In particular, there need not be any correlation between the length of the segment $L_{0}$, which is proportional to $C$, and the pinning strength, which determines $\Delta E$ and $V_{T}$. The recent discovery ${ }^{3}$ that there is a temperature-independent component in the oscillatory conductance suggests that a model of charge-density-wave tunneling like that of Larkin and Lee ${ }^{4}$ might be applicable to this phenomenon, although, again, we do not expect $\Delta E$ and $V_{T}$ to be proportional to $1 / L_{0}$.

Finally, given that the energy scale of the $C B$ is expected to be so large, one might ask why it is not manifested within the charge-density-wave model as well. The reason is that in the latter model no capacitive charging occurs, because the motion of all the electrons in the segment is correlated.

M. A. Kastner, Stuart B. Field, U. Meirav, and J. H. F. Scott-Thomas

Department of Physics

Massachusetts Institute of Technology

Cambridge, Massachusetts 02139

D. A. Antoniadis and H. I. Smith

Department of Electrical Engineering

Massachusetts Institute of Technology

Cambridge, Massachusetts 02139

Received 28 July 1989

PACS numbers: 73.20.Dx, 71.45.Lr, 72.15.Nj

${ }^{1}$ H. van Houten and C. W. J. Beenakker, preceding Comment, Phys. Rev. Lett. 63, 1893 (1989).

2J. H. F. Scott-Thomas, S. B. Field, M. A. Kastner, H. I. Smith, and D. A. Antoniadis, Phys. Rev. Lett. 62, 583 (1989).

${ }^{3}$ U. Meirav, M. A. Kastner, M. Heiblum, and S. J. Wind, Phys. Rev. B 40, 5871 (1989).

${ }^{4}$ A. I. Larkin and P. A. Lee, Phys. Rev. B 17, 1596 (1978). 